\title{
Depletion of insulin-like growth factor 1 receptor increases radiosensitivity in colorectal cancer
}

\author{
Yi Li" ${ }^{1 \#}$, Kui Lu${ }^{2 \#}$, Ben Zhao ${ }^{3}$, Xiaokui Zeng ${ }^{4}$, Shan $\mathrm{Xu}^{5}$, Xin $\mathrm{Ma}^{4,6}$, Yunqing Zhi ${ }^{1,4,7}$ \\ ${ }^{1}$ Department of Gastrointestinal Surgery, the Affiliated Hospital of Qingdao University, Qingdao, China; ${ }^{2}$ Department of Oncology, the Second \\ People's Hospital of Taizhou City, Taizhou, China; ${ }^{3}$ Division of Solid Tumor Translational Oncology, German Cancer Consortium and German \\ Cancer Research Center, DKFZ, Heidelberg, Germany; ${ }^{4}$ Department of Transplantation, University Hospital Essen, University of Duisburg- \\ Essen, Essen, Germany; ${ }^{5}$ Department of Radiotherapy, University Hospital Essen, University of Duisburg-Essen, Essen, Germany; ${ }^{6}$ Department \\ of Nephrology, the First Affiliated Hospital of Chengdu Medical College, Chengdu, China; ${ }^{7}$ Department of Gynecology, Shanghai Changning \\ Maternity and Infant Health Hospital, Shanghai, China \\ Contributions: (I) Conception and design: Y Zhi, Y Li; (II) Administrative support: K Lu, Y Li; (III) Provision of study materials or patients: K Lu, \\ Y Li; (IV) Collection and assembly of data: Y Li, K Lu, X Ma, B Zhao; (V) Data analysis and interpretation: X Ma, B Zhao, X Zeng, S Xu; (VI) \\ Manuscript writing: All authors; (VII) Final approval of manuscript: All authors. \\ \#These authors contributed equally to this work. \\ Correspondence to: Yunqing Zhi; Xin Ma. Hohlweg25, Universitätsklinikum Essen, Hufelandstr.55, 45147 Essen, Germany. \\ Email: yunqing.zhi@stud.uni-due.de; xin.ma@stud.uni-due.de.
}

Background: Although radiation therapy for advanced colorectal cancer (CRC) is very effective in some patients, treatment resistance limits its efficacy. Insulin-like growth factor 1 receptor (IGF1R) can affect tumor responsiveness and sensitivity to radiation in several cancer types. Herein, we studied the underlying function of IGF1R in the resistance of advanced CRC to radiation therapy and the possible use of drugs targeting IGF1R to overcome this resistance in patients with CRC.

Methods: Differences in the expression levels of the IGF1R were assessed in CRC samples from patients who were radiosensitive or radioresistant. Two radio-resistant colorectal cancer cell lines, SW480 and HT29, were selected for in vitro studies, and the involvement of the IGF1R in their radiation resistance was elucidated by suppressing its expression through a targeted siRNA and through the use of a specific IGF1R inhibitor, BMS-754807. We assessed radiosensitivity in these human CRC cells lines by examining their proliferation and colony formation, as well as cell cycle analysis. Activation of the Akt pathway was assessed using western blotting.

Results: Compared with tissues from radiosensitive patients, higher IGF1R expression levels were found in patients with radiation-resistant colorectal cancer, while BMS-754807 had improved radiosensitivity and reversed radiation tolerance in both colorectal cancer cell lines. Pre-treatment with BMS-754807 prior to irradiation inhibited Akt phosphorylation, induced cell cycle arrest, and increased DNA damage. Therefore, the IGF1R contributes to radiation resistance of CRC cells in vitro.

Conclusions: This study supports the notion that the radiosensitivity of radiation-resistant colorectal cancer cells can be enhanced by directly targeting IGF1R expression or activity. Ultimately, the combination of radiotherapy with IGF1R targeted inhibitors could potentially increase its effectiveness in the treatment of advanced colorectal cancer.

Keywords: Colorectal cancer (CRC); radiosensitivity; insulin-like growth factor-1 receptor (IGF1R); IGF1R inhibitor

Submitted May 07, 2020. Accepted for publication Sep 20, 2020.

doi: 10.21037/jgo-20-210

View this article at: http://dx.doi.org/10.21037/jgo-20-210 


\section{Introduction}

Colorectal cancer (CRC) remains a common lethal disease (1). Following surgical treatment, the cure rate is correlated with the overall resection rate (2). However, in patients with more advanced colorectal cancer, the mortality rate is high (3). Generally, most patients with CRC are diagnosed during advanced stages. Differences in genetic factors influence sensitivity to radiation therapy. Although the survival rate in advanced patients is low, radiotherapy remains a significant therapeutic strategy for patients with advanced CRC. Unfortunately, resistance to radiation therapy is a major obstacle to patients' survival.

The upregulation of insulin-like growth factor-1 receptor (IGF1R) has been found to be associated with radiation therapy resistance in several tumor types by affecting cell proliferation, survival, and differentiation $(4,5)$. The first report of the IGF1R's association with radiation resistance came from a study in breast cancer patients that found overexpression of IGF1R to be significantly correlated with tumor recurrence following radiation therapy (6). In laryngeal squamous cell carcinoma insensitive to radiotherapy, IGF1R was also found to be overexpressed in cancer cells compared to adjacent cells (7). Both clinical and basic research have confirmed that targeting the IGF1R enhances radiosensitivity in both in vitro and in vivo cancer models $(8,9)$. Additionally, reducing IGF1R protein level or inhibiting its activity can increase the sensitivity of tumor cells to ionizing radiation, and was found to enhance radiosensitivity by increasing DNA damage in both human and murine prostate cancer cell lines (10). The IGF1R has been extensively studied as a potential target for possible anti-cancer treatment strategy $(11,12)$.

In this study, we evaluated the responses of three CRC cell lines to radiotherapy treatment and identified two cell lines with resistant to radiotherapy. We also examined the effect of ionizing radiation in these cells, with or without BMS-754807, an IGF1R inhibitor, or siRNA-mediated knockdown of the IGF1R. We present the following article in accordance with the MDAR reporting checklist (available at http://dx.doi.org/10.21037/jgo-20-210).

\section{Methods}

\section{Tissue samples}

Sixty-two patients with CRC were enrolled from the colorectal surgery department in our center from August 2013 to December 2016. CRC tissues and normal tissues
$3 \mathrm{~cm}$ from the tumor were collected. Patients' mean age was $56.2 \pm 14.1$ years with no history of other malignancies. Patient underwent an imaging examination and were clinically staged based on the examination results. The stages were as follows: T1 refers to a tumor invades submucosa, T2 refers to a tumor invades the muscularis propria, T3 refers to a tumor invading the subserosal layer, $\mathrm{T} 4$ refers to a tumor invading other organs or structures, N0 refers to no lymph node metastasis, $\mathrm{N} 1$ refers to the presence of less than three local lymph node metastases, M0 refers to no other organ metastasis, and M1 refers to distant organ metastasis. All patients had completed preoperative neoadjuvant radiotherapy prior to surgery, and the biopsy tissue samples were obtained during radical surgery. The study was conducted in accordance with the Declaration of Helsinki (as revised in 2013). The study was approved by the ethics committee of Qingdao University (No. QDFYWZLL25796) and informed consent was taken from all the patients.

\section{Cell lines}

Three human colorectal cancer cell lines (HT29, SW480, and DLD-1) were obtained from American Type Culture Collection (USA). Cell cultures were maintained at $37{ }^{\circ} \mathrm{C}$ in an atmosphere of $5 \% \mathrm{CO}_{2}$. The HT29 and DLD- 1 cells were cultured in Dulbecco's modified Eagle's medium (DMEM) with glucose and L-glutamine. The SW480 cells were cultured in Roswell Park Memorial Institute (1640) medium. All media were supplemented with $1 \%$ penicillin streptomycin (PS) (Sigma, USA) and 10\% fetal bovine serum (FBS) (GIBCO, Australia).

\section{Immunobistochemistry}

Sections of $5 \mu \mathrm{m}$ thickness were prepared from both tumor tissue and normal tissue adjacent to the cancer tissue from each patient. Five visual fields were selected from each slice, and the percentage of positive cancer cells and the staining intensity of tissue were scored for each visual field. Eight fields of view were randomly selected under high magnification. IPP software was used to measure the staining intensity of each field of vision. Mean optic density (MOD) was used as the Semi-quantitative analysis of protein expression.

\section{Irradiation}

Cells were cultured in $6 \mathrm{~cm}$ dishes at a density of 
approximately $1 \times 10^{4}$ cells per dish for overnight, and were irradiated with different doses of ionizing radiation $(2,4$, 6, and 8 Gy), using a Faxitron 43855 F X-ray radiometer (Faxitron, USA). The radiation parameters were $160 \mathrm{kV}$ and $6.3 \mathrm{~mA}$. The durations of exposure for 2, 4, 6, and $8 \mathrm{~Gy}$ of irradiation were $1.1,2.3,3.5$, and 4.6 minutes, respectively. Following irradiation, fresh tissue culture medium was added to the cells by replacing the old one and they were cultured for $0,12,24,36$, and 48 hours.

\section{Colony formation assay}

Cells were cultured in 6-well plates at an optimum density of $1 \times 10^{3}$ cells per well in three individual groups. After cells were cultured for 24 hours, $4 \mu M$ BMS-754807, an IGF$1 \mathrm{R}$ inhibitor, was added to the DMEM medium of the third group of cells, while the first two groups of cells being left untreated. After culturing the cells for 24 hours, the latter two groups were then irradiated with the dose of $2 \mathrm{~Gy}$. The irradiated cells were then returned to the incubator for 12 days post which they were immersed in $1 \%$ crystal violet solution for 15 minutes. The number of colonies with over 20 cells was determined and used to calculate the survival score (SF). The survival scores at 2 Gy (SF2) of irradiation were used to assess radiosensitization.

\section{Western blotting}

Proteins were extracted using a cell lysis kit (Sigma, USA). Cells were solubilized in RIPA lysis buffer and diluted to the same concentration using SDS-PAGE sample loading buffer. Samples containing same weight of protein were separated electrophoretically and semidry transferred to nitrocellulose membrane $(45 \mu \mathrm{m})$. The membrane was blocked using the QuickBlock Blocking Buffer (Sigma, USA) for 20 minutes and then incubated and treated with the appropriate primary antibody at the refrigerator $\left(4^{\circ} \mathrm{C}\right)$ overnight. The nitrocellulose membranes were rinsed in phosphate buffer solution and Tween 20 (PBST) and then incubated with the goat anti-rabbit secondary antibodies (horseradish peroxidase, HRP) for 2 hours. After rinsing again for three times, immunoreactive proteins were detected using SuperSignal West Pico Substrate (Thermo, USA) and automatic scanning. The antibodies in this study were as follows: anti-IGF-1R (Santa Cruz, USA), anti- $\gamma \mathrm{H} 2 \mathrm{AX}$ (Abcam, USA), goat anti-rabbit secondary antibodies (Abcam, USA), and anti- $\beta$-actin (Abcam, USA).

\section{Real-time RT-PCR}

Total RNA was extracted using RNAeasy ${ }^{\mathrm{TM}}$ RNA Isolation Kit (Sigma, USA), and then transcribed into cDNA. The mRNA levels were determined by PCR using a LightCycler480II (Roche, Switzerland) with the levels of $\beta$-actin mRNA used for normalization. The primers used were: 5'-GCTTGCCGCCACTACTACTATGC-3' (forward) and 5'-GAGGTAACAGAGGTCAGCATTTT-3' (reverse) for IGF-1R and 5'-CTTCTACAATGAGCTGCGTGTGG-3' (forward) and 5'- ATGGCTGGGGTGTTGAAGGTGTCA-3' (reverse) for $\beta$-actin.

\section{Transfection of small interfering RNA (siRNA)}

A total of $2 \times 10^{5}$ cells/well were seeded in each well of 6-well plates and incubated for overnight. After 15 hours, the cells were transfected with siRNA using the DharmaFECT 4 transfection reagent (Dharmacon). Cells were then harvested 24 hours post transfection. The sequences of the siRNAs were as follows: IGF-1R siRNA (sense strand: 5'-CGACUAUCAGCAGCUGAAGTT-3', antisense strand 5'-CUUCAGCUGCUGAUAGUCGTT-3'), and control siRNA (sense strand: 5'-GAAGUCGACGACUAUCAGCTT-3', antisense strand: 5'-GCUGAUAGUCGUCGACUUCTT-3').

\section{MTT cell viability assay}

A total of 3,000 cells for testing were cultured in each well of 96-well plates. Next, $20 \mu \mathrm{L}$ of 3-(4,5-dimethylthiazol-2-yl)2,5-diphenyl tetrazolium bromide (MTT, $5 \mathrm{mg} / \mathrm{mL}$ ) was added to each well. After culturing the cells for 4 hours, the medium containing the MTT was discarded, and DMSO was added to the cells. At 0, 12, 24, 36, and 48 hours, the optical density (OD) at $480 \mathrm{~nm}$ was determined using a microplate reader. The percentage of OD on day $n$ divided by the OD on day 0 was used to calculate the cell survival rate. The operation was repeated at least three times.

\section{Cell cycle assay}

A total of $1 \times 10^{5}$ cells/well were cultured in 6-well plates. After culturing overnight, cells were irradiated with 2 Gy of X-rays. After culturing for 48 hours, cell cycle was evaluated. Cells were washed twice with PBS, fixed in $75 \%$ ethanol overnight at $4{ }^{\circ} \mathrm{C}$, then resuspended in $100 \mathrm{uL}$ containing $500 \mathrm{mg} / \mathrm{L}$ RNase Ade PBS, cultured at $37^{\circ} \mathrm{C}$ for 1 hour, then resuspended again in $500 \mathrm{uL}$ PBS containing 


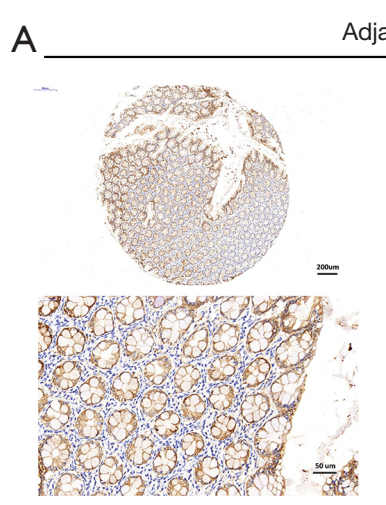

Radiation-insensitive
Adjacent

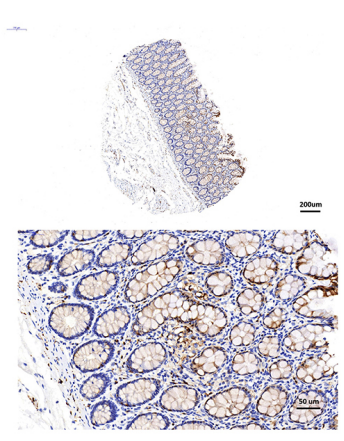

Radiation-sensitive

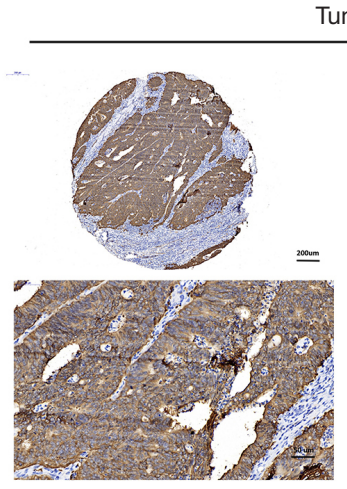

Radiation-insensitive
Tumor

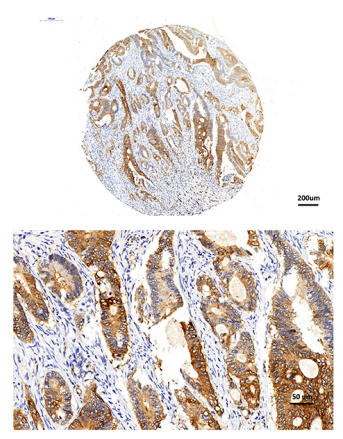

Radiation-sensitive
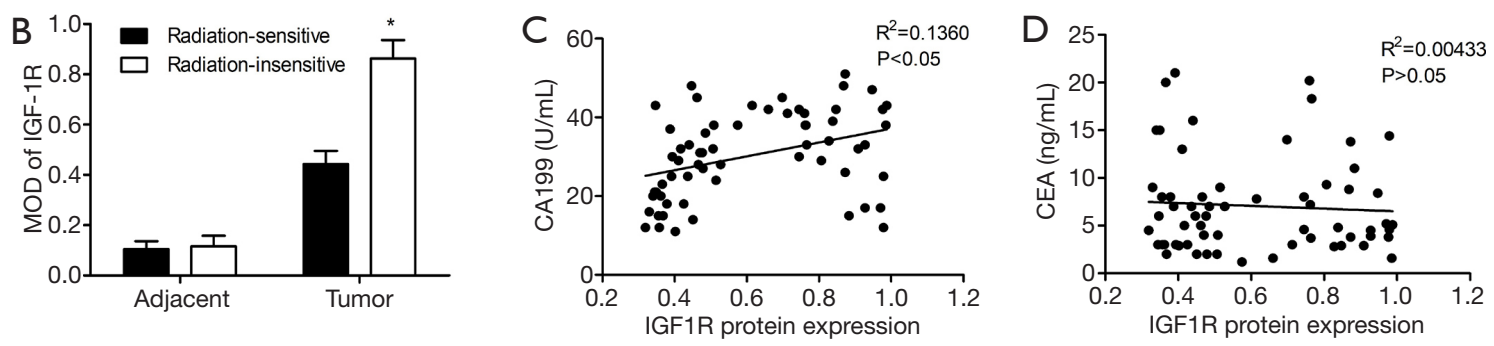

Figure 1 Increased expression of the IGF1R in radiation resistant colorectal cancer tissues. (A,B) Higher expression in the radiationresistant CRC tissues. (C,D) The correlation analysis showed the expression of IGF1R protein from CRC tissues had positive correlation with clinic CA199 values whereas had no obvious correlation with CEA.

$50 \mathrm{mg} / \mathrm{L}$ propidium iodide (PI) for 45 minutes at $4{ }^{\circ} \mathrm{C}$. The proportion in G1, S and G2-M cell cycle phases were recorded using flow cytometry. At least three independent experiments were conducted.

\section{Statistical analysis}

The data are presented as mean \pm standard (SD) by Student's $t$-test for two independent groups or one-way ANOVA for multiple groups. A $\chi^{2}$ test and Tukey's test were used for the analysis of variables. All analyses were performed using SPSS 19.0 software (IBM, NY, USA). A P value of $<0.05$ was considered statistically significant.

\section{Results}

\section{Increased expression of the IGF1R in radiation resistant colorectal cancer tissues}

In this study, we enrolled 62 CRC patients from Qingdao University (25 radiation-insensitive cases and 37 radiation- sensitive cases) from whom we had access to CRC tumor samples, as well as normal tissue adjacent to the tumor. Using immunohistochemical staining, we compared the IGF1R protein expression in the CRC tissues from radiation-sensitive patients with those from radiationinsensitive patients and found higher expression in the radiation-resistant CRC tissues (Figure 1A,B). We compared the IGF-1R protein expression levels in 62 CRC tissue samples with pathological results and clinical indicators. IGF1R protein expression levels were correlated with the radiosensitivity and tumor stage and not significantly correlated with age, sex, or invasion of surrounding tissues of colon cancer patients (Table 1). CA199 and CEA are commonly used clinical tumor biomarkers for colorectal cancer. Correlation analysis showed that the IGF1R protein expression from CRC tissues was positively correlated with clinical CA199 values $(\mathrm{r}=0.2512, \mathrm{P}=0.042)$ and not with CEA $(\mathrm{r}=-0.0978, \mathrm{P}=0.449)$ (Figure $1 C, D)$. These data therefore show that the IGF1R expression levels are positively correlated with tumor radiotherapy resistance, distant metastasis, and lymph node metastasis. 
Table 1 The relationship between IGF1R expression and characteristics of colorectal cancer

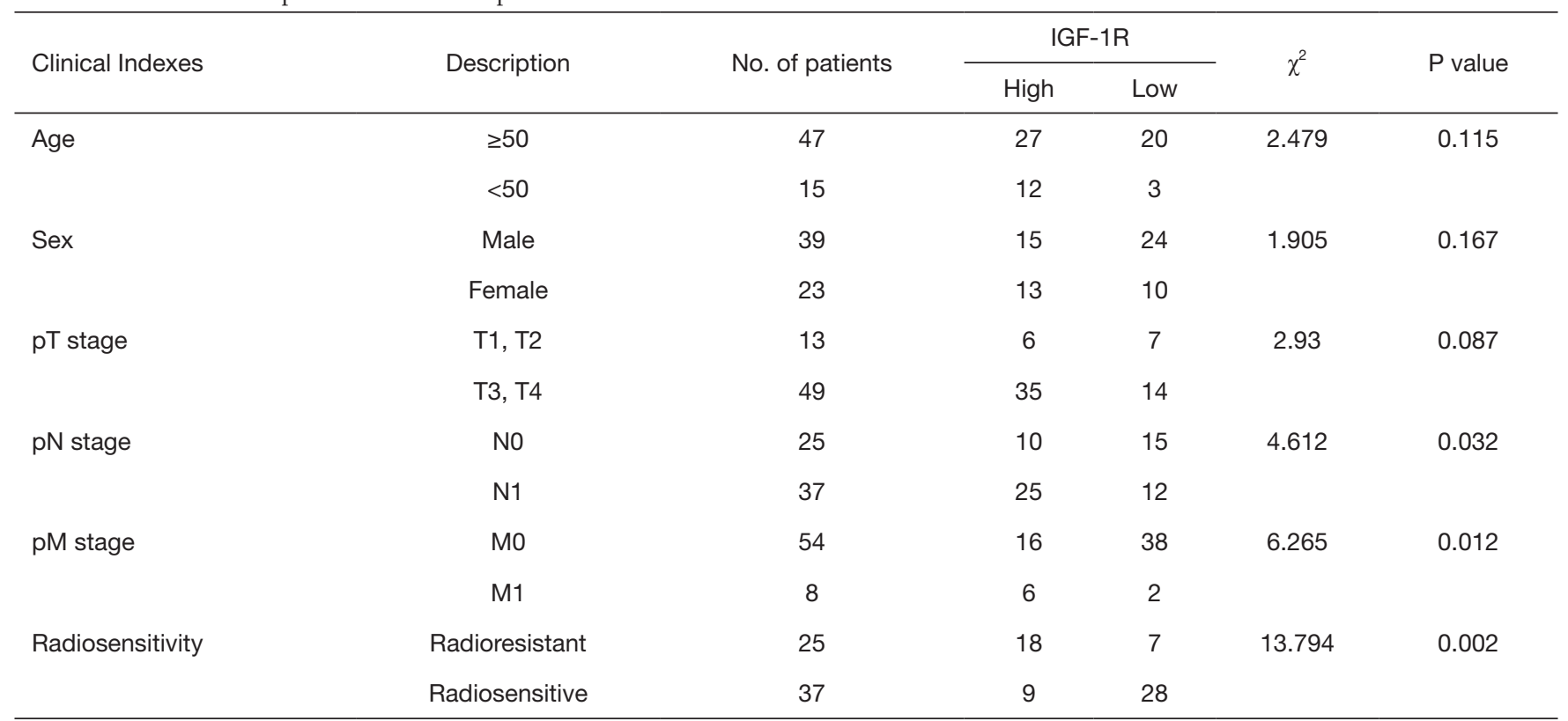

$\mathrm{P}<0.05$ indicated significant differences.

\section{High expression levels of the IGF1R in radiation-resistant CRC cell lines}

RT-PCR was used to measure the IGF1R mRNA in three cell lines, namely HT29, SW480, and DLD-1 cells. We found that among these three cell lines, the IGF1R mRNA levels were high in HT29 and SW480 cells and relatively lower in DLD-1 cells (Figure $2 A$ ). The results showed that the IGF1R protein levels in DLD-1 cells were indeed lower than those seen in SW480 and HT29 cells (Figure 2B). Based on the cell survival score, we predicted that the expression levels of the IGF1R would be positively correlated with radiation resistance $(\mathrm{r}=0.8425, \mathrm{P}<0.0001$ and $\mathrm{r}=0.8120, \mathrm{P}<0.0001$, Figure $2 C, D)$. Based on these data we selected SW480 and HT29 cells as two radiotherapyresistant CRC cell lines that we then used to construct IGF1R knockdown models to further study how IGF1R affects the sensitivity to radiotherapy.

\section{Silencing IGF1R with siRNA or inbibiting it with BMS- 754807 can interfere with the cell cycle in CRC cell lines}

Based on the correlation between radiation resistance and the IGF1R expression, we next explored whether inhibiting the IGF1R expression affects the cell cycle. The level of IGF1R
mRNA can be suppressed by transfecting SW480 and HT29 cells with siRNA specifically targeting IGF1R mRNA (Figure 3A). BMS-754807 also has an inhibitory effect on the mRNA level of IGF1R (Figure 3B).

The SW480 cells with or without knockdown of the IGF1R by siRNA were irradiated with 2 Gy of ionizing radiation and were then cultured for 48 hours. Control groups were not exposed to radiation (0 Gy). In the presence of radiation, compared with radiation alone, we found that siRNA-mediated IGF1R silencing caused an increase in number of G2-M cells with radiation. Similar results were observed in HT29 cells (Figure 3C).

We treated cells with various concentrations of BMS754807 and measured the $\mathrm{IC}_{50}$ of BMS-754807 using dosesurvival curve. $\mathrm{IC}_{50}$ was $2.92 \mu \mathrm{M}$ in SW480 cells treated with BMS-754807 and $3.47 \mu M$ in HT29 cells. The SW480 and HT29 cells with or without treatment of $4 \mu M$ BMS754807 were also irradiated with 2 Gy of ionizing radiation and then cultured for 48 hours. In the presence of radiation, compared with the cells that were not treated with BMS754807, we found that IGF1R knockdown cells treated with BMS-754807 increased G2-M (Figure 3C). Based on these results, irradiation of cells with a knockdown of the IGF1R results in a greater degree of G2/M blockade, thereby potentially increasing the sensitivity to radiotherapy. 

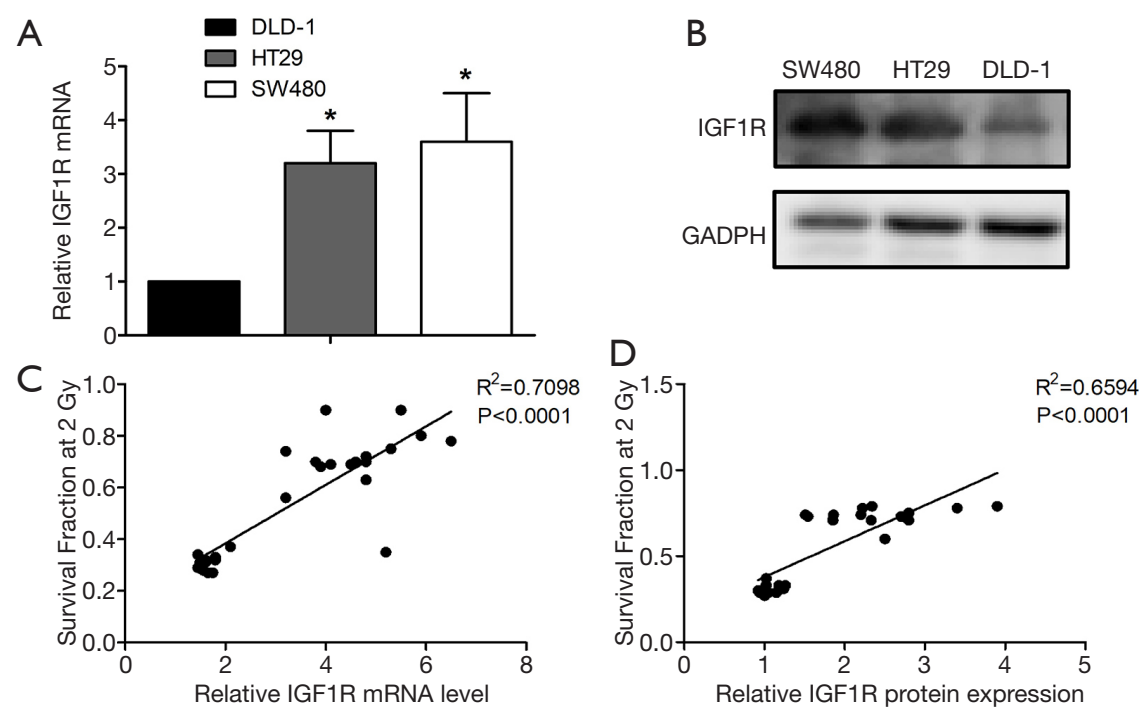

Figure 2 High expression levels of the IGF1R in radiation-resistant CRC cell lines. (A) The IGF1R mRNA levels were high in HT29 and SW480 cells and relatively lower in DLD-1 cells. ${ }^{*} \mathrm{P}<0.05$ vs. DLD-1 cell lines. (B) The IGF1R protein levels in DLD-1 cells were indeed lower than those seen in SW480 and HT29 cells. (C,D) The correlation analysis showed that both the mRNA and protein expression levels of the IGF1R were positively correlated with radiation sensitivity $(\mathrm{r}=0.8425, \mathrm{P}<0.0001$ and $\mathrm{r}=0.8120, \mathrm{P}<0.0001)$.
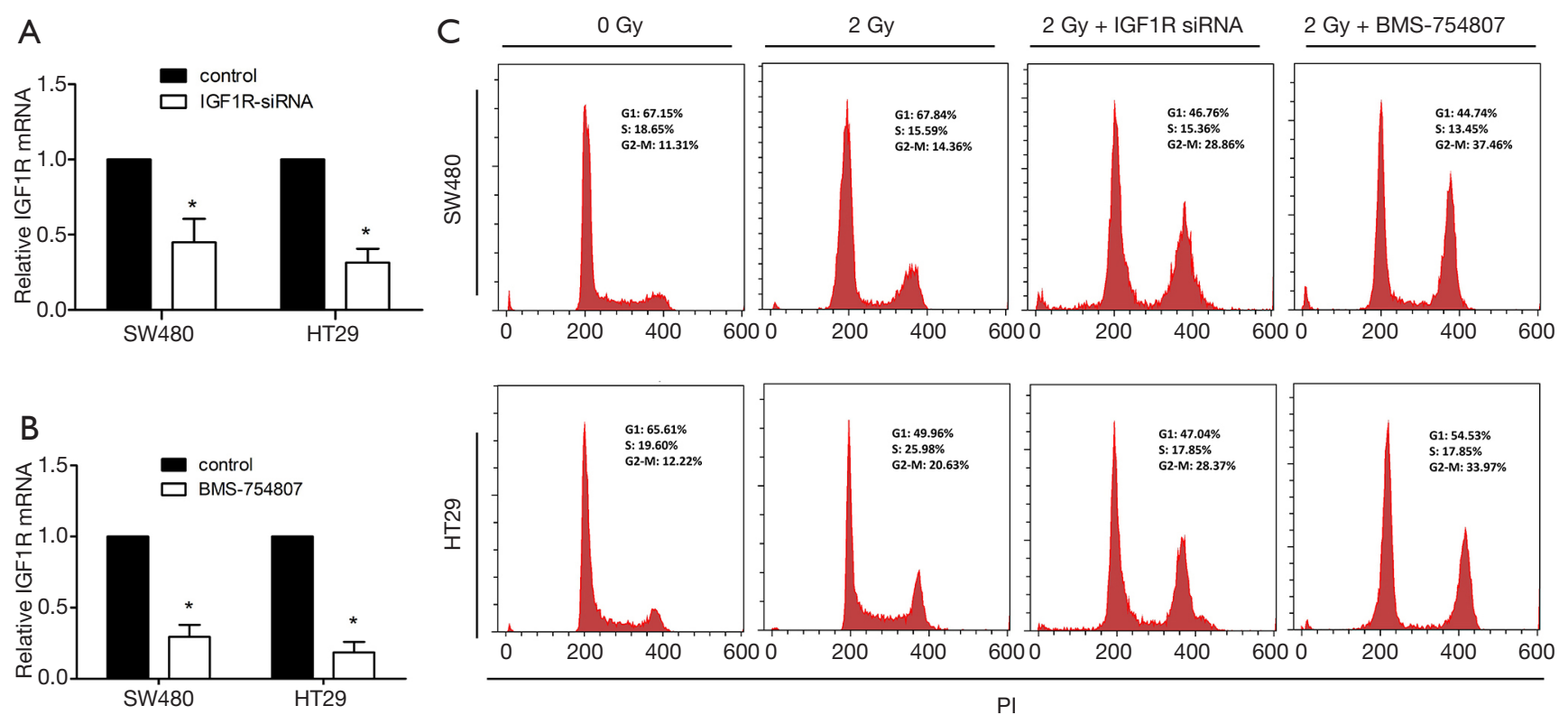

Figure 3 Silencing the IGF1R with siRNA or inhibiting the IGF1R with BMS-754807 can interfere with the cell cycle in CRC cell lines. (A) The mRNA expression of IGF1R in SW480 and HT29 cell lines after siRNA treatment was measured by qRT-PCR. *P<0.05 vs. control. (B) The mRNA expression of IGF1R in SW480 and HT29 cell lines treated with BMS-754807 was measured by qRT-PCR. *P<0.05 vs. control. (C) Knockdown of the IGF1R by siRNA-mediated or treated with BMS-754807 both caused an increase in number of G2-M cells with radiation. 

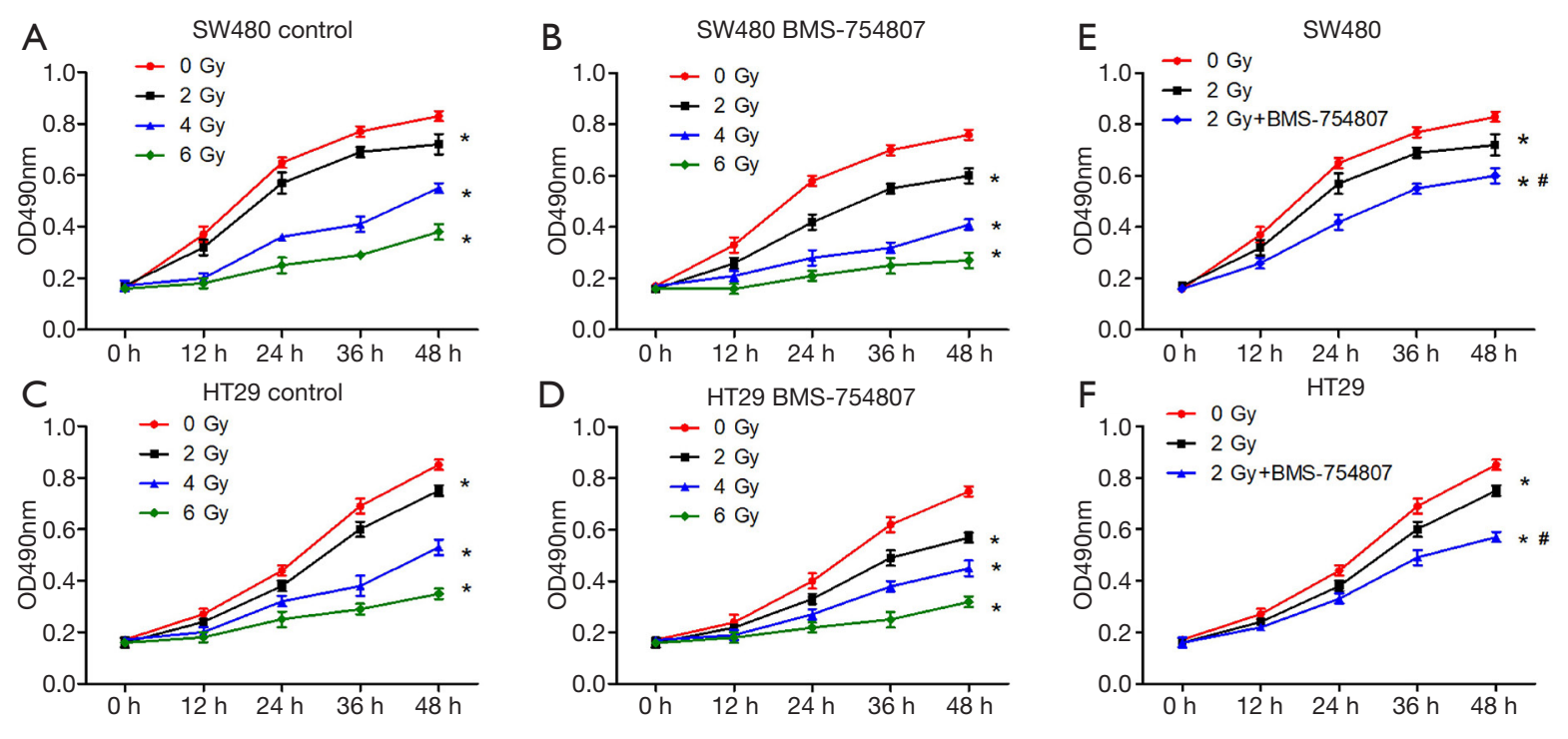

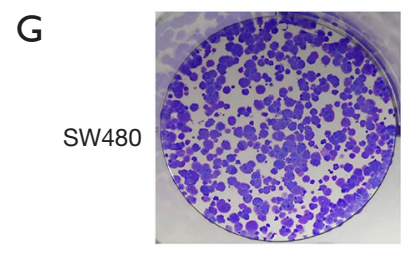

Control

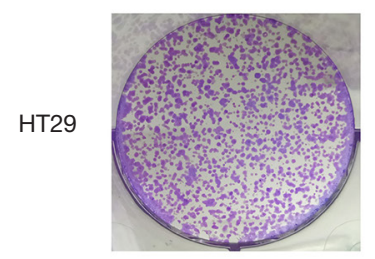

Control

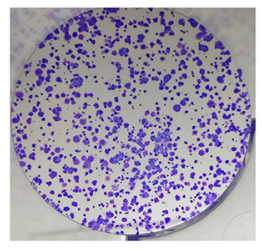

$\mathrm{IR}$

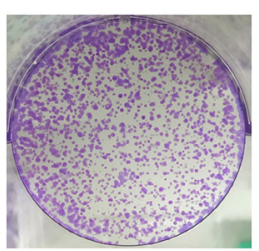

IR

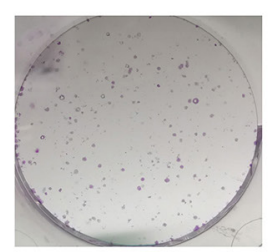

IR + BMS-754807

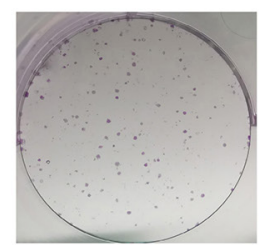

IR + BMS-754807
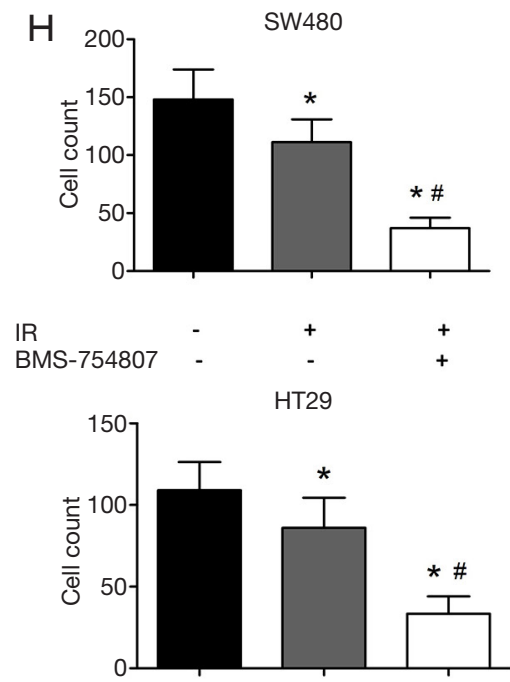

$\begin{array}{llll}\mathrm{IR} & - & + & + \\ \text { BMS-754807 } & - & - & +\end{array}$

Figure 4 BMS-754807 promotes a response in colorectal cancer cells to irradiation. (A,B) Proliferation in SW480 cells treated and untreated with BMS-754807 was significantly reduced in a dose-dependent matter after irradiation. ${ }^{*} \mathrm{P}<0.05$ vs. 0 Gy. (C,D) Similar results were seen in HT29 cells. (E,F) Compared with only 2 Gy radiation treatment group, the proliferation inhibitory effect after combined BMS-754807 was statistically increased. ${ }^{*} \mathrm{P}<0.05$ vs. 0 Gy, ${ }^{\#} \mathrm{P}<0.05$ vs. 2 Gy. $(\mathrm{G}, \mathrm{H})$ The results of colony formation test showed that BMS-754807 could inhibit proliferation in both irradiated SW480 and HT29 cells. ${ }^{*} \mathrm{P}<0.05$ vs. control, ${ }^{*} \mathrm{P}<0.05$ vs. IR.

\section{BMS-754807 promotes a response in colorectal cancer cells to irradiation}

To investigate whether an inhibition of the IGF1R using the inhibitor BMS-754807 affects the radiosensitivity of HT29 and SW480 CRC cells, we treated both cells with or without BMS-754807 and then irradiated them with X-rays, and assessed for colony formation and proliferation. Cells were treated with BMS-754807 and cultured for 24 hours. Experimental group cells were then irradiated with ionizing radiation of 2, 4, or $6 \mathrm{~Gy}$. Cells with or without BMS-754807 treatment were all irradiated, showing an inhibition of cell proliferation in a dose-dependent matter (Figure 4A,B,C,D). SW480 cells proliferation decreased following 2 Gy X-rays irradiation, but when combined with BMS-754807, a more significant proliferation inhibition 

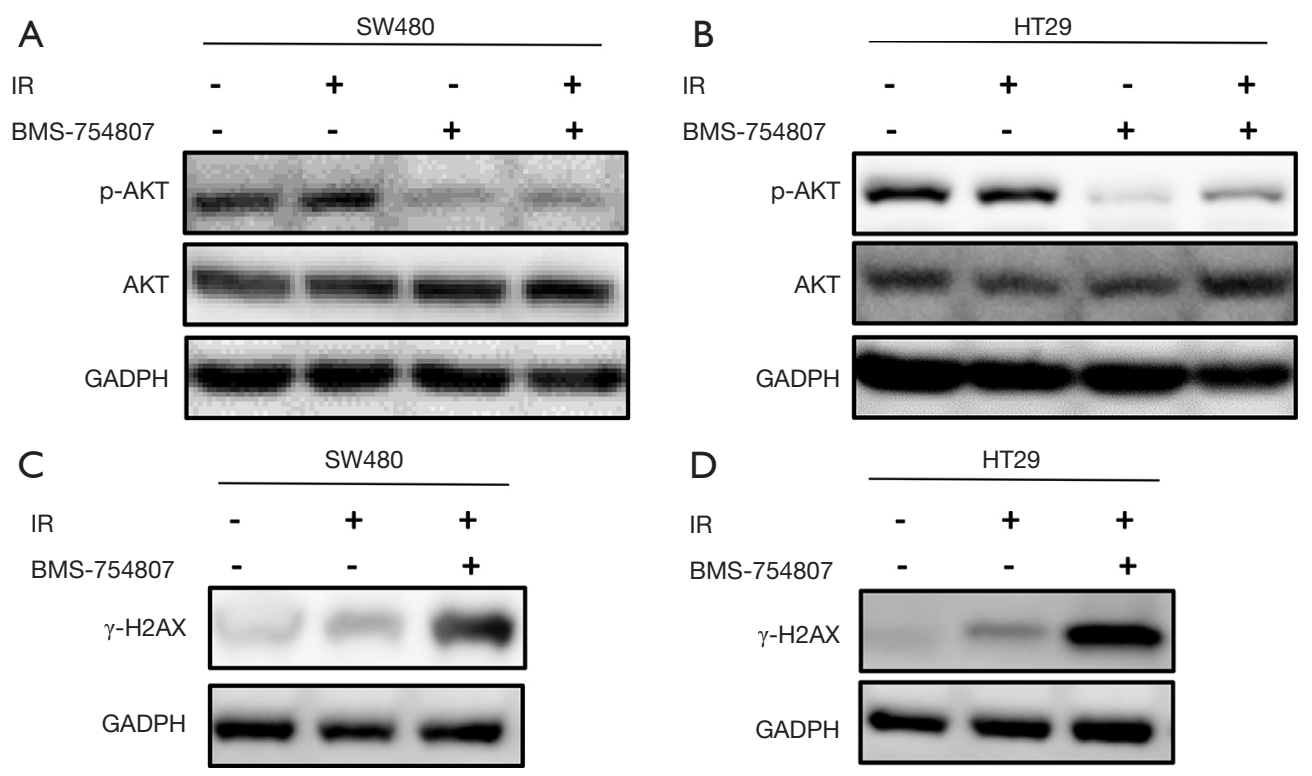

Figure 5 Changes in the Akt phosphorylation signaling pathway and double-stranded DNA breaks. (A) In SW480 cells treated with BMS754807, the phosphorylation levels of Akt reduced followed by irradiation effectively. (B) The HT29 cells showed similar results. (C,D) radiation can increase the formation of the phosphorylated form of $\mathrm{H} 2 \mathrm{AX}(\gamma \mathrm{H} 2 \mathrm{AX})$, while BMS-754807 treatment can further enhance the formation of $\gamma \mathrm{H} 2 \mathrm{AX}$.

was observed (Figure 4E), suggesting that BMS-754807 increases the radiosensitivity of SW480 cells. Similar results were seen in HT29 cells (Figure 4F).

Based on the clear changes in proliferation of BMS754807 treated cells induced by 2 Gy of radiation, we selected the same dose (2 Gy) to treat the cells in subsequent experiments. Colony formation test was used to evaluate the changes in clonogenicity. Crystal violet staining showed, compared with the radiation-only group, BMS-754807 treatment had significantly inhibited the proliferation of SW480 and HT29 cells following irradiation (Figure 4G,H).

\section{Changes in the Akt phosphorylation signaling patbway and double-stranded DNA breaks}

The western blotting analysis showed that in SW480 cells, irradiation with X-rays increased p-Akt levels. SW480 cells treated with BMS-754807 alone showed reduction in p-Akt levels. Treating cells with BMS-754807 followed by irradiation (2 Gy) effectively reduced p-Akt levels in SW480 cells (Figure 5A). The HT29 cells showed similar results (Figure 5B). This indicates that BMS-754807 can block the PI3K/AKT pathway, thereby increasing the sensitivity of radiotherapy.

To analyze the effect of BMS-754807 on double strand
DNA damage following irradiation, we also assessed the phosphorylation levels of $\gamma-\mathrm{H} 2 \mathrm{AX}$. When radiation causes DNA double-strand breaks, H2AX phosphorylation increases according to the degree of DNA damage. Our results showed that radiation can increase the formation of the phosphorylated $\mathrm{H} 2 \mathrm{AX}(\gamma-\mathrm{H} 2 \mathrm{AX})$, while BMS-754807 treatment can further enhance the formation of $\gamma-\mathrm{H} 2 \mathrm{AX}$ (Figure 5C,D). These results indicate that inhibiting IGF1R can increase DNA damage in CRC cell line under radiation exposure, thereby increasing radiosensitivity.

\section{Discussion}

High levels of IGF1R expression are positively correlated with radiation resistance in cervical, prostate, lung, HPVnegative head and neck cancers, and melanoma (10,13-17). Studies have shown that the higher IGF1R expression, the higher the grade of various tumors and the lower the survival rate (18-20). Our study has demonstrated the potential of IGF1R as a high radiosensitizing molecular target in patients with CRC. IGF1R expression level (which may be an indicator of lymphatic metastasis or distant metastasis of CRC) in tumor sites of the radiotherapy resistance group was significantly higher than that in the radiotherapy sensitive group. CEA and CA199 are commonly used clinical tumor 
biomarkers for colorectal cancer. CEA is a broad-spectrum tumor marker, which has important clinical diagnostic and evaluation value of colorectal cancer, while CA199 levels are significantly correlated with lymph node metastasis and pTNM staging (21). The results of this study indicate that the expression level of IGF1R at the tumor site was closely correlated with CA199 serum levels.

By combining clinical patient tissue samples and several in vitro analyses in two radio-resistant human CRC cell lines, we confirmed that the IGF1R expression is correlated with radiotherapy resistance.

Several other in vitro studies have also suggested that increased expression of IGF1R confers radio-resistance to cells $(22,23)$. Furthermore, we found that SW480 and HT29 cell lines, which had reduced levels of IGF1R due to knockout, had reduced survival and an increased sensitivity to radiation, which confirms a role for the IGF1R in the radioresistance seen in CRC. BMS-754807, a reversible ATP competitive IGF1R/IR inhibitor, has been found to inhibit IGF1R phosphorylation and activation of the PI3-K pathway in response to IGF-1 in breast cancer cells (24). In this regard, Akt is commonly activated in most cancers and is correlated with resistance to radiotherapy (25-27). Consistent with these data, we showed that irradiation alone increased the levels of p-AKT in these cell lines, however, decreased following BMS-754807 treatment, suggesting that BMS-754807 increases the sensitivity of colorectal tumors to radiation by inhibiting the radiation-induced activation of Akt. Sensitization to radiotherapy or chemotherapy by BMS-754807 has been previously reported in breast cancer, lung cancer, and pediatric high-grade gliomas $(24,28,29)$. Our study is therefore the first to examine the effect of BMS754807 on radiosensitivity in human CRC cells.

A cell cycle study has confirmed that radiation induced G2-M arrest, while the addition of IGF-1R inhibitor, lincitinib, induces further arrest of nasopharyngeal carcinoma cells in G2-M phase (30). Consistent with these results, our study has demonstrated that BMS-754807 can also arrest G2-M, and that combination of IR and BMS-754807 can effectively enhance the radiosensitivity of CRC cells.

Radiation causes double-strand breaks (DSB), thereby destroying DNA structure (31), which causes ataxia telangiectasia mutant protein (ATM) to be activated, ultimately arresting the cell cycle (24). It is also known that ATM sensing of DNA damage can induce the expression of IGF-1 to drive pro-survival signals following exposure to radiation (32).Upon exposure to ionizing radiation, $\mathrm{H} 2 \mathrm{AX}$ is phosphorylated, while each DSB can form a constant amount
ofy-H2AX. For a 1 Gy X-ray dose, approximately 1\%-2\% of H2AX is phosphorylated intoy-H2AX $(9,33)$. After DNA damage in the cell, H2AX is phosphorylated. The increased levels of $\gamma \mathrm{H} 2 \mathrm{AX}$ seen following treatment with BMS754807 would likely increase the sensitivity of tumor cells to radiation, which is consistent with the data observed. Similar results were found in both SW480 and HT29 cells. Consistent with pervious findings, our study proved that IGF1R inhibitor treatment in the presence of radiation can significantly increase DNA damage in tumor cells (34). It has also been shown that BRCA2 can help to reduce DNA damage through homologous recombination, whereas a kinase-deficient IGF1R has been shown to inhibit BRCA2 expression (35). These results further indicate that inhibiting IGF1R activity may increase the radiosensitivity of CRC. Finally, IGF1R can affect nonhomologous end-joining thus protecting cells from radiation-induced DNA damage (36).

In conclusion, we report that high IGF1R expression levels are associated with adverse tumor responses to radiation in patients with colorectal cancer and can reduce the radiosensitivity of colorectal cancer cells. BMS-754807, an IGF1R inhibitor, can increase DNA damage, downregulate the PI3K/Akt pathway, and increase G2-M phase in cell cycle all of which can increase radiosensitivity and reverse radioresistance in colorectal cancer. In the future, we intend to conduct further studies, both animal and clinical, to verify that BMS-754807 treatment can overcome radiotherapy resistance.

\section{Acknowledgments}

Funding: This study was funded by Special scientific research project of the First Affiliated Hospital of Chengdu Medical College (CYFY16DL-02).

\section{Footnote}

Reporting Checklist: The authors have completed the MDAR reporting checklist. Available at http://dx.doi.org/10.21037/ jgo-20-210

Data Sharing Statement: Available at http://dx.doi. org/10.21037/jgo-20-210

Peer Review File: Available at http://dx.doi.org/10.21037/ jgo-20-210

Conflicts of Interest: All authors have completed the ICMJE 
uniform disclosure form (available at http://dx.doi. org/10.21037/jgo-20-210). The authors have no conflicts of interest to declare

Ethical Statement: The authors are accountable for all aspects of the work in ensuring that questions related to the accuracy or integrity of any part of the work are appropriately investigated and resolved. The study was conducted in accordance with the Declaration of Helsinki (as revised in 2013). The study was approved by the ethics committee of Qingdao University (No. QDFYWZLL25796) and informed consent was taken from all the patients.

Open Access Statement: This is an Open Access article distributed in accordance with the Creative Commons Attribution-NonCommercial-NoDerivs 4.0 International License (CC BY-NC-ND 4.0), which permits the noncommercial replication and distribution of the article with the strict proviso that no changes or edits are made and the original work is properly cited (including links to both the formal publication through the relevant DOI and the license). See: https://creativecommons.org/licenses/by-nc-nd/4.0/.

\section{References}

1. 1. Siegel RL, Miller KD, Goding Sauer A, et al. Colorectal cancer statistics, 2020. CA Cancer J Clin 2020;70:145-64.

2. Silva GL, de Moura EG, Bernardo WM, et al. Endoscopic versus surgical resection for early colorectal cancer-a systematic review and meta-analysis. J Gastrointest Oncol 2016;7:326-35.

3. Edge SB, Compton CC. The American Joint Committee on Cancer: the 7th edition of the AJCC cancer staging manual and the future of TNM. Ann Surg Oncol 2010;17:1471-4.

4. Ryan PD, Goss PE. The emerging role of the insulin-like growth factor pathway as a therapeutic target in cancer. Oncologist 2008;13:16-24.

5. Gennigens C, Menetrier-Caux C, Droz JP. Insulin-Like Growth Factor (IGF) family and prostate cancer. Crit Rev Oncol Hematol 2006;58:124-45.

6. Turner BC, Haffty BG, Narayanan L, et al. Insulin-like growth factor-I receptor overexpression mediates cellular radioresistance and local breast cancer recurrence after lumpectomy and radiation. Cancer Res 1997;57:3079-83.

7. Qureishi A, Rieunier G, Shah KA, et al. Radioresistant laryngeal cancers upregulate type 1 IGF receptor and exhibit increased cellular dependence on IGF and EGF signalling. Clin Otolaryngol 2019;44:1026-36.

8. Isebaert SF, Swinnen JV, McBride WH, et al. Insulin-like growth factor-type 1 receptor inhibitor NVP-AEW541 enhances radiosensitivity of PTEN wild-type but not PTEN-deficient human prostate cancer cells. Int J Radiat Oncol Biol Phys 2011;81:239-47.

9. Riesterer O, Yang Q, Raju U, et al. Combination of antiIGF-1R antibody A12 and ionizing radiation in upper respiratory tract cancers. Int J Radiat Oncol Biol Phys 2011;79:1179-87.

10. Chitnis MM, Lodhia KA, Aleksic T, et al. IGF-1R inhibition enhances radiosensitivity and delays doublestrand break repair by both non-homologous endjoining and homologous recombination. Oncogene 2014;33:5262-73.

11. Xu Y, Kong GK, Menting JG, et al. How ligand binds to the type 1 insulin-like growth factor receptor. Nat Commun 2018;9:821.

12. Pollak M. The insulin receptor/insulin-like growth factor receptor family as a therapeutic target in oncology. Clin Cancer Res 2012;18:40-50.

13. Ramcharan R, Aleksic T, Kamdoum WP, et al. IGF$1 \mathrm{R}$ inhibition induces schedule-dependent sensitization of human melanoma to temozolomide. Oncotarget 2015;6:39877-90.

14. Lloret M, Lara PC, Bordón E, et al. IGF-1R expression in localized cervical carcinoma patients treated by radiochemotherapy. Gynecol Oncol 2007;106:8-11.

15. Dale OT, Aleksic T, Shah KA, et al. IGF-1R expression is associated with HPV-negative status and adverse survival in head and neck squamous cell cancer. Carcinogenesis 2015;36:648-55.

16. Aleksic T, Verrill C, Bryant RJ, et al. IGF-1R associates with adverse outcomes after radical radiotherapy for prostate cancer. Br J Cancer 2017;117:1600-6.

17. Liu X, Chen $\mathrm{H}, \mathrm{Xu} X$, et al. Insulin-like growth factor-1 receptor knockdown enhances radiosensitivity via the HIF-1 $\alpha$ pathway and attenuates ATM/H2AX/53BP1 DNA repair activation in human lung squamous carcinoma cells. Oncol Lett 2018;16:1332-40.

18. Ferté C, Loriot Y, Clémenson C, et al. IGF-1R targeting increases the antitumor effects of DNA-damaging agents in SCLC model: an opportunity to increase the efficacy of standard therapy. Mol Cancer Ther 2013;12:1213-22.

19. Moreno-Acosta P, Gamboa O, Sanchez de Gomez M, et al. IGF1R gene expression as a predictive marker of response to ionizing radiation for patients with locally 
advanced HPV16-positive cervical cancer. Anticancer Res 2012;32:4319-25.

20. Valsecchi ME, McDonald M, Brody JR, et al. Epidermal growth factor receptor and insulinlike growth factor 1 receptor expression predict poor survival in pancreatic ductal adenocarcinoma. Cancer 2012;118:3484-93.

21. Hai Luo, Kexin Shen, Bo Li, et al. Clinical significance and diagnostic value of serum NSE, CEA, CA19-9, CA125 and CA242 levels in colorectal cancer. Oncol Lett 2020;20:742-50.

22. Henríquez-Hernández LA, Lloret $M$, Pinar B, et al. BCL-2, in combination with MVP and IGF-1R expression, improves prediction of clinical outcome in complete response cervical carcinoma patients treated by radiochemotherapy. Gynecol Oncol 2011;122:585-9.

23. Osuka S, Sampetrean O, Shimizu T, et al. IGF1 receptor signaling regulates adaptive radioprotection in glioma stem cells. Stem Cells 2013;31:627-40.

24. O'Flanagan CH, O'Shea S, Lyons A, et al. IGF$1 \mathrm{R}$ inhibition sensitizes breast cancer cells to ATMrelated kinase (ATR) inhibitor and cisplatin. Oncotarget 2016;7:56826-41.

25. Han MW, Ryu IS, Lee JC, et al. Phosphorylation of PI3K regulatory subunit $\mathrm{p} 85$ contributes to resistance against PI3K inhibitors in radioresistant head and neck cancer. Oral Oncol 2018;78:56-63.

26. Xu S, Li Y, Lu Y, et al. LZTS2 inhibits PI3K/AKT activation and radioresistance in nasopharyngeal carcinoma by interacting with p85. Cancer Lett 2018;420:38-48.

27. Chen YA, Tzeng DTW, Huang YP, et al. Antrocin Sensitizes Prostate Cancer Cells to Radiotherapy through Inhibiting PI3K/AKT and MAPK Signaling Pathways. Cancers (Basel) 2018;11:34.

Cite this article as: $\mathrm{Li} \mathrm{Y,} \mathrm{Lu} \mathrm{K,} \mathrm{Zhao} \mathrm{B,} \mathrm{Zeng} \mathrm{X,} \mathrm{Xu} \mathrm{S,}$ Ma X, Zhi Y. Depletion of insulin-like growth factor 1 receptor increases radiosensitivity in colorectal cancer. J Gastrointest Oncol 2020;11(6):1135-1145. doi: 10.21037/jgo-20-210
28. Franks SE, Jones RA, Briah R, et al. BMS-754807 is cytotoxic to non-small cell lung cancer cells and enhances the effects of platinum chemotherapeutics in the human lung cancer cell line A549. BMC Res Notes 2016;9:134.

29. Simpson AD, Soo YWJ, Rieunier G, et al. Type 1 IGF receptor associates with adverse outcome and cellular radioresistance in paediatric high-grade glioma. Br J Cancer 2020;122:624-9.

30. Wang Z, Liu G, Mao J, et al. IGF-1R Inhibition Suppresses Cell Proliferation and Increases Radiosensitivity in Nasopharyngeal Carcinoma Cells. Mediators Inflamm 2019;2019:5497467.

31. Mladenov E, Saha J, Iliakis G. Processing-Challenges Generated by Clusters of DNA Double-Strand Breaks Underpin Increased Effectiveness of High-LET Radiation and Chromothripsis. Adv Exp Med Biol 2018;1044:149-68.

32. Goetz EM, Shankar B, Zou Y, et al. ATM-dependent IGF-1 induction regulates secretory clusterin expression after DNA damage and in genetic instability. Oncogene 2011;30:3745-54.

33. Kuo LJ, Yang LX. Gamma-H2AX - a novel biomarker for DNA double-strand breaks. In Vivo 2008;22:305-9.

34. Jun DW, Jeong YS, Kim HJ, et al. Characterization of DDRI-18 (3,3'-(1H,3'H-5,5'-bibenzo[d]imidazole-2,2'-diyl) dianiline), a novel small molecule inhibitor modulating the DNA damage response. Br J Pharmacol 2012;167:141-50.

35. Venkatachalam S, Mettler E, Fottner C, et al. The impact of the IGF-1 system of cancer cells on radiation response An in vitro study. Clin Transl Radiat Oncol 2017;7:1-8.

36. Valenciano A, Henríquez-Hernández LA, Moreno M, et al. Role of IGF-1 receptor in radiation response. Transl Oncol 2012;5:1-9. 